\title{
Retooling Fitness for Noisy Problems in a Supervised Michigan-style Learning Classifier System
}

\author{
Ryan J. Urbanowicz \\ University of Pennsylvania \\ 423 Guardian Dr. \\ Philadelphia, PA \\ 19104-6021,USA \\ ryanurb@upenn.edu
}

\author{
Jason $\mathrm{H}$. Moore \\ University of Pennsylvania \\ 423 Guardian Dr. \\ Philadelphia, PA \\ 19104-6021,USA \\ jhmoore@upenn.edu
}

\begin{abstract}
An accuracy-based rule fitness is a hallmark of most modern Michigan-style learning classifier systems (LCS), a powerful, flexible, and largely interpretable class of machine learners. However, rule-fitness based solely on accuracy is not ideal for identifying 'optimal' rules in supervised learning. This is particularly true for noisy problem domains where perfect rule accuracy essentially guarantees over-fitting. Rule fitness based on accuracy alone is unreliable for reflecting the global 'value' of a given rule since rule accuracy is based on a subset of the training instances. While moderate over-fitting may not dramatically hinder LCS classification or prediction performance, the interpretability of the solution is likely to suffer. Additionally, over-fitting can impede algorithm learning efficiency and leads to a larger number of rules being required to capture relationships. The present study seeks to develop an intuitive multi-objective fitness function that will encourage the discovery, preservation, and identification of 'optimal' rules through accuracy, correct coverage of training data, and the prior probability of the specified attribute states and class expressed by a given rule. We demonstrate the advantages of our proposed fitness by implementing it into the ExSTraCS algorithm and performing evaluations over a large spectrum of complex, noisy, simulated datasets.
\end{abstract}

\section{Categories and Subject Descriptors}

J.3 [Computer Applications]:

Life and Medical Sciences[biology and genetics]

\section{Keywords}

classifier systems, multi-objective optimization, bioinformatics, pattern recognition and classification, multiple solutions

\section{INTRODUCTION}

Michigan-style learning classifier systems (LCS) offer a

Permission to make digital or hard copies of part or all of this work for personal or classroom use is granted without fee provided that copies are not made or distributed for profit or commercial advantage and that copies bear this notice and the full citation on the first page. Copyrights for third-party components of this work must be honored. For all other uses, contact the owner/author(s).

GECCO '15 July 11-15, 2015, Madrid, Spain

(C) 2015 Copyright held by the owner/author(s).

ACM ISBN 978-1-4503-3472-3/15/07.

DOI: http://dx.doi.org/10.1145/2739480.2754756 uniquely powerful and flexible approach to prediction, data mining, classification, and function approximation problems. One of the most important advantages of such rule-based algorithms is that they make few to no assumptions about the underlying pattern or patterns of association in the dataset. This is mainly due to how LCS distributes a learned solution over a population of human interpretable IF:THEN rules [16]. Previously, we developed an extended supervised tracking and classifying system (ExSTraCS) [13]. ExSTraCS is an LCS specialized to single-step supervised learning problems, a large category of problems that includes biomedical applications to which we have a particular interest in addressing (i.e. epidemiological and bioinformatic data mining and prediction). ExSTraCS sacrifices the ability to function on reinforcement learning problems in order to bolster performance in supervised learning domains with particular attention paid to learning efficiency, solution interpretability, and knowledge discovery in problems where the signal may be either clean or noisy. Real world applications such as those found in bioinformatics are typically noisy and may have a large number of potentially predictive attributes, complex patterns of association including epistasis (i.e. non-linear attribute interactions) and heterogeneity (i.e. independent associations with the same class/phenotypic endpoint).

More recently, ExSTraCS 2.0 was introduced, offering a new strategy to dramatically improve the scalability of Michigan-style LCS algorithms [17]. As a demonstration of this, ExSTraCS 2.0 solved the extremely complex 135bit multiplexer problem 'directly' for the first time reported in the literature. This problem solution requires 135 predictive attributes involved in 128 heterogeneous 8-attribute epistatic interactions to predict the binary class of any instance with $100 \%$ accuracy. For a rough conceptual comparison of difficulty, consider that in the field of genetic epidemiology, researchers rarely even attempt to search for epistatic interactions that involve more than 2 or 3 attributes. Despite this success, specific performance drawbacks were identified in ExSTraCS 2.0 related to characterizing heterogeneous patterns of association in noisy simulated bioinformatics problems. It was proposed that this shortcoming could be attributed to the applied fitness metric. Specifically in modern Michigan-style LCS algorithms, fitness is generally based solely on rule accuracy, where (1) for supervised learning, accuracy refers to the proportion of 'matched' training instances for which the rule included the correct classification, and (2) for reinforcement learning, accuracy is a function of a rule's reward prediction error. Rule-accuracy is not 
to be confused with training or testing accuracy which would be computed using the entire training or testing datasets.

The problem with a fitness metric based only on accuracy, is that an individual rule has little concept of what it means to be 'fit' in the context of the rule-population as a whole. Rule accuracy is a 'local' metric of rule value in that it only indicates value within the context of the subset of instances in the dataset that the rule matches. Rule accuracy does not necessarily indicate whether a rule is capturing useful generalizations in the dataset. For instance consider the situation where a rule matches only a single instance in the training dataset, and correctly classifies that instance. This rule would have a perfect accuracy. However, this rule likely represents an over-fit relationship identified in the training set that will have no useful predictive value in a testing dataset. In a clean problem, such as the standard multiplexer problems, implicit and explicit generalization pressures would help to eliminate such overfit rules in favor of equally accurate, but more general ones. This is not the case in noisy problems. Consider that in a noisy dataset, optimal rules can not achieve perfect rule accuracy. Instead, rules that are over-fit (i.e. additionally specify non-predictive attributes) will dominate, since overfit rules are likely to have accuracies closer to 1 . When it comes to knowledge discovery within the rule population by means of manual rule inspection (i.e. identifying individual rules that capture the learned patterns of association), neither accuracy nor accuracy-based fitness can be used to rank the rule-population to identify the best rules. Instead, rule numerosity (i.e. the number of copies of a given rule stored in the rule population), serves as the only reasonable guide for ranking and identifying the best rules [19]. However, numerosity merely captures 'rule-value' indirectly and thus is not entirely reliable [9].

The strategy of 'fitness sharing' was implemented by Wilson as part of XCS in order to give individual rules a broader context of value [19]. Specifically, fitness sharing proportionally adjusts fitness so that rules with the highest accuracy within individual niches of the problem have the highest fitness. Bernado-Mansilla expanded their supervised learning LCS, called UCS [2] to include fitness sharing [7], noting some of it's performance trade-offs in supervised learning problems. In particular, fitness sharing was valuable at improving performance in class imbalance problems. However, fitness sharing still relies exclusively on accuracy, which we would expect to remain problematic in noisy problem domains, where the multi-objective balance between accuracy, and maximally (correctly) covering instances in the training data is critical to a global measure of rule value.

The most significant change to how rule fitness has been calculated in Michigan-style LCS algorithms involved the shift from strength-based fitness in ZCS [18] to accuracybased fitness in XCS [19], proposed primarily in the context of reinforcement learning problems. Strength-based fitness relied on the predicted payoff that would be received when a given rule's action was chosen. However, accuracy-based fitness valued the accuracy of the payoff prediction rather than it's magnitude. This was a key to niching performance, allowing the algorithm to focus on and retain more than the strongest parts of the solution [4]. The development of UCS [2] included a new definition for accuracy-based rule fitness. In this supervised learning context, rule accuracy was simply the proportion of instances for which a rule predicted the correct class out of all those that it matched, and fitness was determined by a simple power function of this accuracy. This was the accuracy-based rule fitness adopted by ExSTraCS.

Since XCS, there have been relatively few attempts to develop an alternate fitness metric. Within Michigan-style LCSs, a class-sensitive accuracy/fitness metric was proposed to deal with class imbalance [6], a Bayesian classifier system was proposed to deal with noisy binary class problems [3], and a mutual information-based fitness developed for supervised learning and the discovery of default hierarchies [8] were all developed yielding mixed results. Within Pittsburgh-style LCSs there is no rule-level fitness, rather fitness is calculated at the level of the rule-set. Fitness research for this class of algorithms focused on the problem of balancing rule-set accuracy with parsimony (i.e. rulegeneralization and set size). One Pittsburgh-style study proposed multi-objective learning classifier systems, and considered the use of a Pareto-front to better deal with noisy problems [5]. Notably a Pareto-front is typically intended for comparing complete solutions. Individual LCS rules do not constitute complete solutions therefore, as is, this approach does not appear to be applicable to Michigan-style LCS algorithms. However, this may make an interesting direction for future work. Another Pittsburgh-style study successfully developed a fitness metric that explicitly combined accuracy and the complexity of a rule set to promote generalization and reduce bloat. [1].

In the present study we propose a new multi-objective approach for calculating rule-level fitness for supervised learning Michigan-style LCS algorithm that combines (1) rule accuracy, (2) correct instance coverage, and (3) an adjustment for the combined state and class occurrence probabilities within a rule. We expect that the inclusion of instance coverage will help overcome the challenge of identifying heterogeneous and epistatic patterns in the context of noisy data by reflecting the value of having rules that are both accurate, and that are correctly applicable to a maximal number of instances. The adjustment for occurrence probabilities give rules that specify rare state combinations and/or rare classes (in the case of imbalanced data) a fitness boost to account for expected lower correct rule coverage. We believe that the development of a globally-minded fitness metric will not only speed up the effective discovery of optimal rules, but improve rule population interpretability, and the overall process of knowledge discovery. This proposed fitness strategy has been implemented and tested within the ExSTraCS 2.0 algorithm and evaluated over a spectrum of complex, noisy simulated genetic datasets concurrently modeling epistatic and heterogeneous patterns of association. We expect this new fitness metric to (1) eliminate the interpretability shortcomings previously identified in ExSTraCS 2.0 [17], (2) to further improve both global and manual knowledge discovery, and (3) promote more efficient learning. Additionally, we implemented and tested fitness-sharing as described in [7] to observe potential performance trade-offs in the context of the ExSTraCS algorithm and demonstrate that this mechanism alone is insufficient to address the problem of comparing global rule-value.

\section{METHODS}

In this section we briefly introduce (1) the ExSTraCS 2.0 algorithm along with some minor alterations designed to accommodate the new fitness metric, (2) our implementation of fitness-sharing (3) the multi-objective fitness metric with 
discussion of the logic behind the components, and (3) the simulated datasets and evaluation strategy applied to test this proposed fitness metric.

\subsection{Algorithm}

The ExSTraCS 2.0 algorithm [17] is a rule-based machine learning algorithm (or more specifically a Michigan-style LCS algorithm) that has been expanded and adapted to better suit the needs of real-world supervised learning problems wherein classification, prediction, data mining, and/or knowledge discovery is the goal. It is descended from a lineage of Michigan-style LCSs founded primarily on the architecture of XCS [19], the best-studied LCS to date. In descending chronological order, precursors of ExSTraCS 2.0 include ExSTraCS 1.0 [13], EK-UCS [14], AF-UCS [11], UCS [2], and XCS [19]. The features of ExSTraCS 2.0 that most differentiate it from UCS or XCS include: a rule specificity limit to address scalability issues [17], built-in rapid expert knowledge (EK) generation algorithms [13], EK guided covering and mutation for efficient learning and scalability [14, 17], attribute tracking and feedback for reusing useful attribute combinations and characterizing patterns of heterogeneity [11], built-in rule compaction strategies [9], and the consolidation of explore/exploit to perform simultaneously [12]. For a detailed description of the ExSTraCS 2.0 algorithm see [17]. For a complete software users guide see [10]. In this study the ExSTraCS 2.0 algorithm with our proposed multi-objective fitness will be referred to as ExSTraCS 2.1. Each version is available on sourceforge.com.

ExSTraCS 2.1 inherits all of the mechanisms and run parameters applied to ExSTraCS 2.0 [17] except for the following elements that have been updated to better suit the proposed multi-objective fitness strategy. First, we have eliminated class/endpoint mutation within the GA in order to ensure that all rules introduced to the rule-population match and correctly classify the current training instance. As a result of this, all rules now start out with a perfect fitness (i.e. fitness $=1$ ) as they are initialized with perfect accuracy. As you will see in the next section, rules are also initialized with a maximum correct instance coverage. Second, once a given rule has had the opportunity to 'see' the entire training set we label that rule as 'epoch-complete'. Once this happens, the accuracy of that rule becomes fixed, and does not continue to change with experience, as any other fluctuations are the result of the random order of the training set. This brings consistency to how experience, or 'epochcomplete' rules are compared to one another through the fitness calculation. Lastly, to accommodate the constantly changing fitness estimate of 'inexperienced' rules introduced below we recalculate the fitness of every 'epoch-incomplete' rule whenever the matching phase occurs.

\subsection{Fitness Sharing}

We implemented fitness sharing into ExSTraCS 2.0 as described in [7], where this strategy had previously been implemented into UCS to evaluate it's effect within a supervised learning environment. In summary, rule-accuracy is calculated as previously described in ExSTraCS 2.0 [17], however fitness is no longer a simple power function of accuracy. Instead, whenever a match set is formed, a new accuracy metric $k_{r}$ is first determined. If rule accuracy $\left(A_{r}\right)$ is above some user defined threshold $\left(A_{0}\right)$ than $k_{r}=1$, otherwise:

$$
k_{r}=\alpha\left(A_{r} / A_{0}\right)^{\nu}
$$

As in [7], $\alpha=0.1, A_{0}=0.999$, and we examine a value of 1 and 10 for $\nu$. Next we calculated the relative accuracy $\left(k_{r}^{\prime}\right)$ of the rule to be the numerosity weighted ratio of $k_{r}$ to the sum of all $k_{r}$ in the current match set. Lastly, rule fitness is updated using the following equation:

$$
F_{r}=F_{r}+\beta\left(k_{r}^{\prime}-F_{r}\right)
$$

In this way, rules that consistently have the highest accuracy within the match sets that it participates, will obtain the highest fitness values. We have implemented this into the original ExSTraCS 2.0 algorithm, as well as some test implementations combined with our proposed multiobjective fitness metric described below.

\subsection{Multi-objective Fitness}

In this section we detail the proposed multi-objective fitness metric. It includes three components; (1) an adjusted accuracy, (2) an adjusted ratio of correct instance coverage, and (3) an adjustment for a rule's combined state and class occurrence probabilities.

\subsubsection{Accuracy Component}

First we calculate the accuracy component $\left(A c_{r}\right)$. As we only want to value rule accuracy $\left(A_{r}\right)$ that is greater than expected by random chance, we compare accuracy to the probability of picking the rule's class by chance $\left(p_{c}\right) . p_{c}$ need only be calculated once during the pre-processing phase of ExSTraCS. Using $p_{c}$ rather than a constant accounts for class imbalance or the possibility of multiple class problems. If $A_{r}>p_{c}$ than a term we call useful accuracy $\left(A u_{r}\right)$ is calculated as:

$$
A u_{r}=A_{r}-p_{c}
$$

Otherwise one of two alternative $A u_{r}$ calculations are applied. In the special case where a rule is still relatively young (i.e. it has only had the opportunity to see less than 1000 learning iterations), and the second instance it matched happened yielded an incorrect classification, we do not want the rule to be assigned too low a value of $A u_{r}$. In order to protect young rules from having their accuracy cut in half on the second match of it's existence, useful accuracy is calculated as $A u_{r}=p_{c} / 2$. We have somewhat arbitrarily chosen 1000 iterations as as reasonable rule-age cutoff (AgeLimit $)_{r}$. If $A_{r} \leq p_{c}$ and this special case did not occur, than $A u_{r}=A_{r} / 1000$. This yields a very small accuracy component. Next we scale $A u_{r}$ so that it will range from 0-1 by dividing it by the maximum possible $A u_{r}$ for a rule with this class (i.e. $1-p_{c}$ ). Lastly we transform this scaled useful accuracy $\left(A s u_{r}\right)$ using the following exponential function so that increases in $A s u_{r}$ increase fitness with the greatest magnitude when closer to random class selection, than they are when they are close to perfect accuracy.

$$
A c_{r}=2 * \frac{\left(\left(1 /\left(1+\exp \left(-5 * A s u_{r}\right)\right)\right)-0.5\right)}{0.98661429815}
$$

This function yields $A c_{r}$ values between 0 to 1 . The utilization of this transformation was inspired by our previous observation applying a higher $\nu$ to the fitness power function 
of accuracy led to worse performance in noisy problems [12]. This is due to the fact that at larger values of $\nu$, any accuracy closer to 1 has a much heavier influence on fitness than a lower accuracy, leading to dramatic over-fitting in noisy problems. While it has been suggested that lowering the user specified parameter $A_{0}$, could help manage this problem in noisy domains, optimally setting this value is heavily problem dependent and would require either prior knowledge of the signal to noise ratio, or a potentially extensive and computationally expensive parameter sweep.

\subsubsection{Correct Coverage Component}

Next, we calculate the correct instance coverage component $\left(C c_{r}\right)$. Intuitively, we want to try to discover and preserve rules that capture an optimal balance of accuracy and instance coverage. However, if $A_{r}$ was less than or equal to $p_{c}$ (i.e. rule accuracy was lower than randomly choosing the correct class), we skip both the coverage and state probability adjustment described hereafter, and the final fitness only relies on $A c_{r}$. This will encourage the deletion of such rules by the algorithm.

The correct instance coverage refers to the number of training instances that have been correctly covered by the current rule. In preliminary work we explored a number of terms introduced into the fitness function in an attempt to give a boost to rules that were more general, and thus correctly covered more instances. For instance, we considered rule specificity, match coverage, and correct coverage and found that they all end up overvaluing rules with low specificity at the expense of accuracy. Another challenge to consider when including coverage, is that as a rule's specificity increases, there is an expected reduction in the probability of a rule to be able to cover a given instance. Also, by not accounting for matching or correct covering based on prior probabilities, such coverage terms tended to yield misleading fitness metrics.

As a result, we came up with a term we call 'useful coverage' $\left(C r_{r}\right)$, which is essentially the coverage of a rule observed beyond what is expected by random chance. This is calculated similar to $A u_{r}$, and in-fact, the $C u_{r}$ term is somewhat of a combination of coverage and accuracy, since it's magnitude relies, in part, on $A_{r}$. $C u_{r}$ is calculated as:

$$
C u_{r}=C c_{r}-\left(C m_{r} * p_{c}\right)
$$

where $C c_{r}$ is the correct coverage of the rule and $C m_{r}$ is the match coverage of the rule (i.e. the number of instances this rule matches in the training set). However, using raw coverage would be problematic, as it would make it harder for high order interactions to be detected, since they naturally will have a lower coverage due to compounding state probabilities. Therefore, we convert $C u_{r}$ into a coverage ratio $C r_{r}$ based on the largest $C u_{r}$ we have yet observed during training $\left(C u_{E c \max }\right)$, where Ecmax is the maximum for rules that have completed an epoch.

$$
C r_{r}=C u_{r} / C u_{E \max }
$$

Note that a separate Ecmax is stored and updated for each class to account for potential class imbalance. Also note, that we store a separate maximum useful coverage for rules that have not completed an epoch $\left(C u_{\text {Eimax }}\right)$. Note that every time fitness is calculated we check to see if either $C u_{\text {Ecmax }}$ or $C u_{\text {Eimax }}$ needs to be updated. This is where labeling of rules as epoch complete or incomplete becomes particularly important. Fundamentally, when training on a finite dataset, as is the assumption when using ExSTraCS, a point is reached where two groups of rules will exist in the population; i.e. those that have seen the entire dataset (epoch-complete), and those that have not, (epochincomplete). Once a rule has seen the entire dataset, accuracy is fixed and we can now directly compare such a rule with all other epoch-complete rules. In LCSs with a fitness based only on accuracy, comparing rule fitness's that are experienced vs. inexperienced is not a problem since a rule can have a very high accuracy from the start. However, when it comes to coverage, there can be a serious discrepancy between rules that are epoch-complete and those that are not. This unfairly biases experienced rules, such that epoch-incomplete rules may not survive long enough to see all the training instances and demonstrate their value.

To account for this in $C u_{r}$, we adopt an extrapolation strategy to estimate $C u_{r}$ for an epoch-incomplete rule. If $C u_{r}$ is younger than this rule we extrapolate $C u_{r}$ up to the age of the current $C u_{\text {Eimax }}$ as follows:

$$
\text { Cuex }_{r}=C u_{r} * A g e_{\text {Eimax }} / A g e_{r}
$$

where Age Eimax is the age of the epoch-incomplete maximum useful coverage, and $A g e_{r}$ is the age of the current rule. If $A g e_{r}>A g e_{\text {Eimax }}$ we extrapolate $C u_{\text {Eimax }}$ as follows:

$$
\text { Cuex } x_{\text {Eimax }}=C U_{\text {Eimax }} * A g e_{r} / A g e_{\text {Eimax }}
$$

In either case the larger of the two $C u$ values is set to $C u_{\text {Eimax }}$, and $A g e_{\text {Eimax }}$ is left at the older age to which the extrapolation was performed. However, we do not allow very young rules to impact the value of $C u_{\text {Eimax }}$. A rule must have Age $_{r}>$ AgeLimit . $_{\text {. }}$.

As with epoch-complete rules, we convert $C u_{r}$ into a coverage ratio $C r_{r}$ based on $C u_{\text {Eimax }}$ as follows:

$$
C r_{r}=C u_{r} / C u_{\text {Eimax }}
$$

\subsubsection{State/Class Probability Correction}

Lastly, to obtain $C c_{r}$ we add an adjustment for the rule's combined state and class occurrence probabilities $\left(S c p_{r}\right)$. First note that during the pre-processing state of ExSTraCS, we initially calculate and store the state frequencies for every discrete attribute in the dataset. For each rule we calculate $S c p_{r}$ to be the product of all specified attribute state frequencies and $p_{c}$ for the class given by that rule. Once this is calculated we check to see if $S c p_{r}$ is the maximum or minimum among rules that have the same specificity (i.e. we separately store max and min $S c p_{r}$ for rules with the same number of specified attributes. This helps to account for inherently smaller $S c p_{r}$ values as rule specificity increases. Next, we calculate the adjustment as follows:

$$
S c A d j_{r}=1-\left(\left(S c p_{r}-S c p_{\min }\right) / S c p_{\max }\right)
$$

Keep in mind that the minimum and maximum $S c p_{r}$ are for rules with the same specificity as the the current rule and that $S c A d j_{r}$ can have a value between 0 and 1 . The idea here is that rarer state/class occurrence probabilities will yield and adjustment near 1 resulting in a fitness boost, while those that are maximally common will not impact fitness at 
all, leaving the $C c_{r}$ entirely up to correct coverage. Lastly we calculate $C c_{r}$ as follows:

$$
C c_{r}=C r_{r}+\left(1-C r_{r}\right) * S c A d j_{r} * S c W e i g h t
$$

where $S c W$ eight $=0.5$. This new fitness constant indicates the fraction of coverage component that can be recovered by the $S c A d j_{r}$ term. If this value was set to 1 , the rarest state/class combinations would always have an optimal correct coverage term (which would end up over-valuing rare state/class combinations.) This parameter might be tuned to improve performance on different problem domains.

We complete our calculation of rule fitness $\left(F_{r}\right)$ to be an equally weighted combination of the average of the accuracy and correct coverage components.

$$
F_{r}=\left(A c_{r}+C c_{r}\right) / 2
$$

\subsection{Evaluation}

It was previously demonstrated that ExSTraCS 2.0 outperformed ExSTraCS 1.0 [17], which in turn had improved performance over AF-UCS and EK-UCS [13], which both had improved performance over XCS and UCS on similar complex noisy simulated genetic problems $[12,14,11]$. In the present study we compare and evaluate ExSTraCS with and without fitness sharing, as well as with and without the proposed multiobjective fitness function. Each implementations was run over the same set of 960 noisy (i.e. heritabilities of $0.1,0.2$, or 0.4 ), complex simulated genetic datasets with 20 discrete-valued attributes that were described and applied in $[13,17]$ and generated using GAMETES [15]. Each dataset concurrently modeled patterns of epistasis and heterogeneity where four of the attributes were predictive and 16 were non-predictive. 20 replicates of each dataset were analyzed and 10-fold cross validation (CV) was employed to measure average testing accuracy and account for overfitting. ExSTraCS was run up to 200,000 learning iterations but performance was also evaluated after only 10,000 iterations. Pair-wise statistical comparisons were made using the Wilcoxon signed-rank tests. All statistical evaluations were completed using $\mathrm{R}$. Comparisons were considered to be significant at $p \leq 0.05$. All analyses were performed using 'Discovery', a 2400 core Linux cluster available to the Dartmouth research community.

Comparisons were performed over a set of key performance metrics. Accuracy metrics were calculated as a respective 'balanced accuracy' to account for any imbalanced datasets. 'Both Power' is the ability to correctly identify both two-locus heterogeneous models. 'Single Power' is the ability to have found at least one. 'Co-occur. Power' indicates the ability to detect the correct heterogeneous pattern. Generality refers to classifier generality, or the average proportion of unspecified attributes across the classifier population. Macro Population refers to the number of unique classifiers in the classifier population. These metrics are described in greater detail in [17].

\section{RESULTS AND DISCUSSION}

We begin with a brief examination of fitness sharing incorporated into ExSTraCS. In previous work we implemented ExSTraCS with a fitness metric most similar to the one described in UCS [2]. A later version of UCS incorporating

\begin{tabular}{|c|c|c|c|}
\hline \multicolumn{4}{|c|}{200,000 Iterations $+Q R F$} \\
\hline \multirow{2}{*}{$\begin{array}{l}\text { Performance } \\
\text { Statistics }\end{array}$} & \multicolumn{3}{|c|}{$\operatorname{ExSTraCS}(\nu=10)$} \\
\hline & Original & Fitness Sharing & $p$ \\
\hline Train Accuracy & .7882 & .9170 & 个**** \\
\hline Test Accuracy & .6178 & .5907 & *** \\
\hline Both Power & .4052 & .3115 & $\downarrow * * *$ \\
\hline Single Power & .7635 & .6563 & $* * *$ \\
\hline Both Co-Power & .0438 & .0250 & $\downarrow *$ \\
\hline Single Co-Power & .7771 & .7042 & $\downarrow * * *$ \\
\hline Rule Generality & .8026 & .7901 & **** \\
\hline Macro Population & 987.12 & 701.17 & $\downarrow * * *$ \\
\hline Run Time (min) & 63.24 & 44.34 & $\downarrow * * *$ \\
\hline $\begin{array}{ll}- & \text { No significant ch } \\
* & \mathrm{p}<0.05 \text { (Directi } \\
* * & \mathrm{p}<0.001 \\
* * * & \mathrm{p}<<0.001\end{array}$ & & (rows) & \\
\hline
\end{tabular}

Table 1: ExSTraCS 2.0: Original vs. Fitness Share

fitness sharing yielded some problem-specific advantages [7]. Table 1 summarizes the different performance metrics averaged over all 960 analyzed datasets (each run to times for cross validation) calculated after 200,000 learning iterations for ExSTraCS 2.0 with and without fitness sharing $(\nu=10)$. Overall, with the addition of fitness sharing, we observe significant decreases in average testing accuracy, rule generality, and all power metrics. A similar comparison was performed using fitness sharing $(\nu=1)$. The results are not included here, since the performance metrics yielded similar or significantly worse performance than fitness sharing with $\nu=10$. Ultimately fitness sharing dramatically increased over-fitting within this particularly noisy problem domain, leading to the loss in predictive performance, as well as a large loss in solution interpretability. It seems that fitness sharing only exacerbated the accuracy-based fitness drawback within noisy problems.

Now we revert back to ExSTraCS 2.0 without fitness sharing and introduce our proposed multi-objective fitness. Table 2 summarizes the different performance metrics averaged over all 960 analyzed datasets (each run 10 times for cross validation) calculated after 10,000 and 200,000 learning iterations for ExSTraCS 2.0 without and with the proposed multiobjective fitness. Overall, with the addition of the multiobjective fitness function, we observe significant increases in average testing accuracy, Single Power, Both Co-Power, Single Co-Power, Macro-Population Size, and generality after 200,000 learning iterations. No significant change in the Both Power metric was observed. Significant overall decreases were observed for both training accuracy and run time. The observed increase in testing accuracy and decrease in training accuracy indicates an overall reduction in overfitting. As expected, the new fitness function is able to dramatically improve our ability to characterize heterogeneous patterns, evidenced by large significant increases in Both Co-Power. This is achieved while still managing to maintain or improve other critical performance metrics; namely testing accuracy and the three other power metrics. This supports the claim made previously in [17] that identified the loss in Both Co-Power to be a natural drawback to accuracy-based fitness in noisy problem domains. Also, in Table 1, note that after only 10,000 learning iterations the new fitness has largely and significantly outperformed the original fitness on all metrics with the exception of Both Power and run time. This suggests that the proposed multi- 


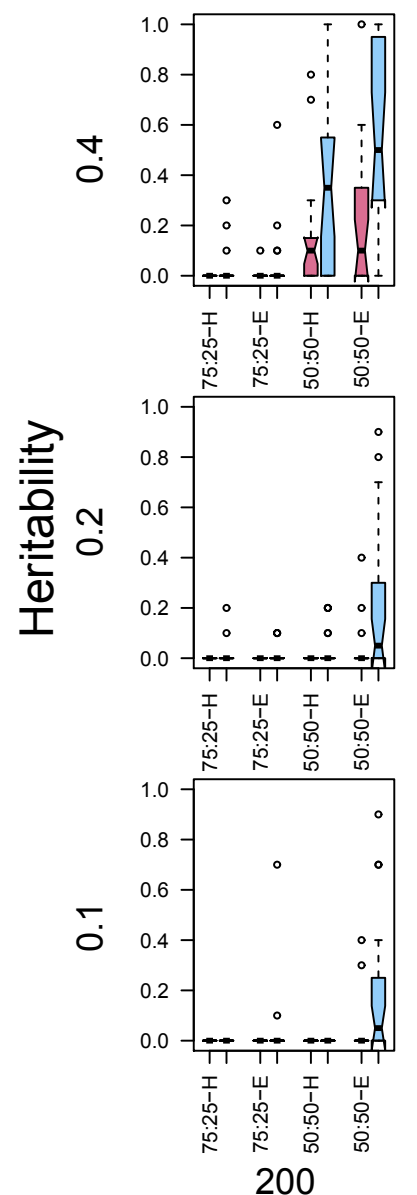

$\square$ Original Fitness $\square$ Multiobjective Fitness
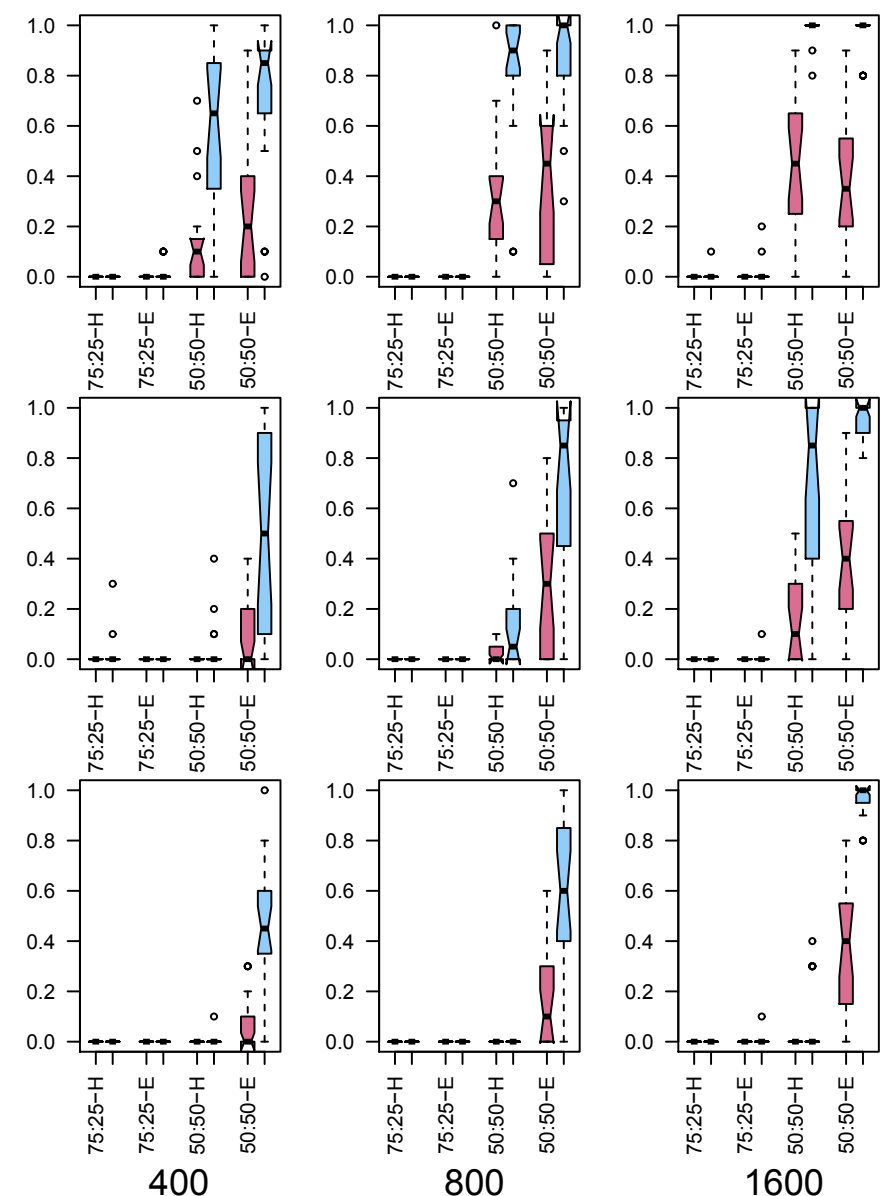

\section{Sample Size}

Figure 1: Comparing Co-Both Power after 200,000 iterations over all dataset combinations in the simulation study.

\section{REFERENCES}

[1] J. Bacardit and J. M. Garrell. Bloat control and generalization pressure using the minimum description length principle for a pittsburgh approach learning classifier system. In Learning Classifier Systems, pages 59-79. Springer, 2007.

[2] E. Bernadó-Mansilla and J. M. Garrell-Guiu. Accuracy-based learning classifier systems: models, analysis and applications to classification tasks. Evolutionary Computation, 11(3):209-238, 2003.

[3] H. Dam, H. Abbass, and C. Lokan. Bcs: a bayesian learning classifier system. Technical report, Citeseer, 2006.

[4] T. Kovacs. Strength or accuracy? Fitness calculation in learning classifier systems. Springer, 2000.

[5] X. Llorà and D. E. Goldberg. Bounding the effect of noise in multiobjective learning classifier systems. Evolutionary Computation, 11(3):279-298, 2003.

[6] A. Orriols and E. Bernadó-Mansilla. Class imbalance problem in ucs classifier system: fitness adaptation. In Evolutionary Computation, 2005. The 2005 IEEE Congress on, volume 1, pages 604-611. IEEE, 2005.
[7] A. Orriols-Puig and E. Bernadó-Mansilla. Revisiting ucs: Description, fitness sharing, and comparison with xcs. In Learning Classifier Systems, pages 96-116. Springer, 2008.

[8] R. E. Smith, M. K. Jiang, J. Bacardit, M. Stout, N. Krasnogor, and J. D. Hirst. A learning classifier system with mutual-information-based fitness. Evolutionary Intelligence, 3(1):31-50, 2010.

[9] J. Tan, J. Moore, and R. Urbanowicz. Rapid rule compaction strategies for global knowledge discovery in a supervised learning classifier system. In Advances in Artificial Life, ECAL, volume 12, pages 110-117, 2013.

[10] R. Urbanowicz. ExSTraCS 2.1, 2015 (accessed February 4, 2015).

[11] R. Urbanowicz, A. Granizo-Mackenzie, and J. Moore. Instance-linked attribute tracking and feedback for michigan-style supervised learning classifier systems. In Proceedings of the fourteenth international conference on Genetic and evolutionary computation conference, pages 927-934. ACM, 2012.

[12] R. Urbanowicz and J. Moore. The application of 

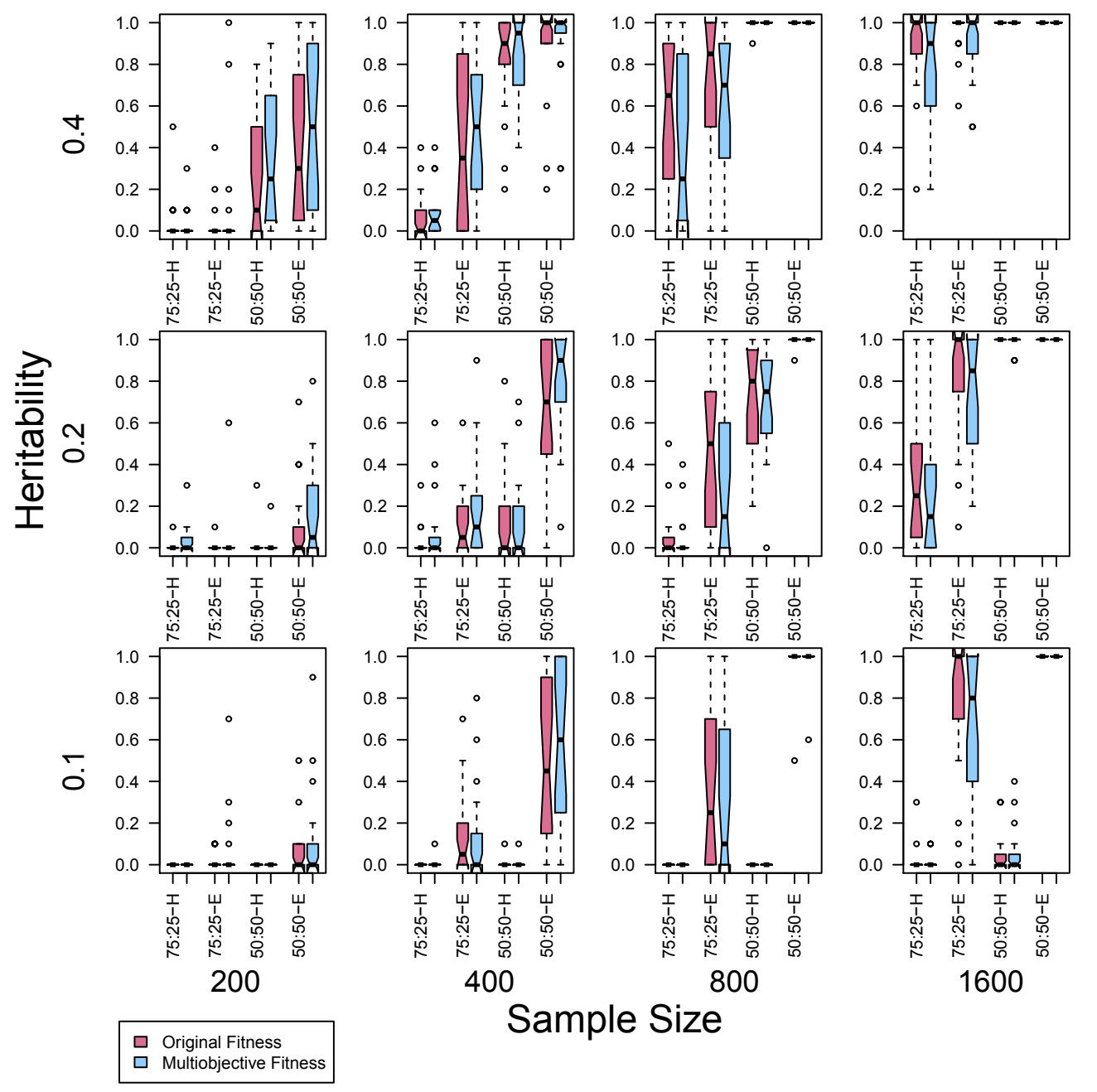

Figure 2: Comparing Both Power after 200,000 iterations over all dataset combinations in the simulation study.

michigan-style learning classifier systems to address genetic heterogeneity and epistasis in association studies. In Proceedings of the 12th annual conference on Genetic and evolutionary computation, pages 195-202. ACM, 2010.

[13] R. J. Urbanowicz, G. Bertasius, and J. H. Moore. An extended michigan-style learning classifier system for flexible supervised learning, classification, and data mining. In Parallel Problem Solving from Nature-PPSN XIII, pages 211-221. Springer, 2014.

[14] R. J. Urbanowicz, D. Granizo-Mackenzie, and J. H. Moore. Using expert knowledge to guide covering and mutation in a michigan style learning classifier system to detect epistasis and heterogeneity. In Parallel Problem Solving from Nature-PPSN XII, pages 266-275. Springer, 2012.

[15] R. J. Urbanowicz, J. Kiralis, N. A.

Sinnott-Armstrong, T. Heberling, J. M. Fisher, and J. H. Moore. Gametes: a fast, direct algorithm for generating pure, strict, epistatic models with random architectures. BioData mining, 5(1):16, 2012.

[16] R. J. Urbanowicz and J. H. Moore. Learning classifier systems: a complete introduction, review, and roadmap. Journal of Artificial Evolution and Applications, 2009.

[17] R. J. Urbanowicz and J. H. Moore. Exstracs 2.0: description and evaluation of a scalable learning classifier system. Evolutionary Intelligence, pages $1-28,2015$.

[18] S. W. Wilson. Zcs: A zeroth level classifier system. Evolutionary computation, 2(1):1-18, 1994.

[19] S. W. Wilson. Classifier fitness based on accuracy. Evolutionary computation, 3(2):149-175, 1995. 